\title{
Research on Decision Methods for Technical Solutions Based on Grey-correlation degree to Vapor Recovery of Refined Oil Terminals
}

\author{
Yuanming Jia ${ }^{1}$, Yiying Zhou ${ }^{1}$, Hongmei Deng ${ }^{1}$, Jing li $^{{ }^{1}}$ \\ ${ }^{1}$ China Waterborne Transport Research Institute, Beijing, 100088, P.R. China
}

\begin{abstract}
In the process of decision on technical solution to vapor recovery of refined oil terminals, the greycorrelation analysis (GCA) is introduced to optimise technical solutions by building a multi-target decision model and using the sequencing of weighted grey-correlation degree (GCD) of evaluation solution as judgment criteria, to determine the priorities of solutions, and the effectiveness of the decision method is verified by a practical example.
\end{abstract}

\section{Introduction}

Massive escape of vapor do not only pose risks to terminal operations in terms of safety and environment, but also waste resources because light oils such as gasoline, naphtha and aviation kerosene will flee due to vapor evaporation during their loading, and the dynamic change of vapor is nearly visible when the temperature is high in summer. The Law on Prevention and Control of Atmospheric Pollution amended in 2015 specifies that "oil or gas storage tank farms, petrol or gas stations, terminals for crude or refined oils, crude or refined oil vessels, and oil or gas tank trucks, among others, shall be equipped with vapor recovery devices which shall be under normal operation in accordance with applicable national rules and regulations"[1]. So that vapor recovery technology does not only become a typical demonstration to mitigate environmental pollution at terminals, but also is escalated to the technical measures required in national laws.

Adsorption, absorption, condensation, membrane separation and low-temperature catalytic oxidation are several vapor recovery treatment technologies that are currently widely used in terminals. As the State and authorities increase the stand limits on emission concentration of vapor, the environmental requirement cannot be met by using one single treatment technology alone, so processes combinations by coupling several unit treatment technologies are gaining wide applications at terminals. At present, though there are lots of documents concerning comparison of shortcomings between different technical solutions, terminals are yet lack of systemic guidance in deciding their solutions. Based on the Greycorrelation degree(GCD) theory, this paper establishes a multi-target decision mode with the weighted GCD of technical solutions as the criterion to realize the comprehensive evaluation and optimization of technical solutions, and verifies the feasibility and effectiveness of the method through practical application. It provides a scientific decision method for refined oil terminals to choose the optimal technical solution of vapor recovery.

\section{Establishment of Decision Model}

In choosing the technical solution on vapor recovery, it is essential for terminals to consider multiple factors comprehensively, for instance, the reliability and safety of the vapor recovery system, cost of construction and operation, type of cargoes, flowing rate and vapor concentration, and the operational mode etc., which jointly decide the operational outcome of the chosen technical solution. As such, for decision of technical solutions on vapor recovery, it is not a good idea to evaluate and select technical solutions just based on a single index alone, instead, it will make the result more accurate and objective by building a decision model to comprehensively evaluate technical solutions with multilevels and multi-targets.

\subsection{Determination of Decision Matrix[2]}

2.1.1 Decision solution set. Suppose, in multi-target decision problems, there are $\mathrm{m}$ solutions to be evaluated, and the evaluation solution set is $\mathrm{U}=\left\{\mathrm{u}_{1}, \mathrm{u}_{2}, \ldots \mathrm{u}_{m}\right\}$, and according to the research, the process combinations of vapor recovery which are more applied at terminals include adsorption - absorption ( Ads.-Abs. ) , condensation - absorption (Con.-Abs.) , membraneseparation-based combinations (absorption - membrane and condensation - membrane separation, Abs-M.S.\& Con.-M.S.) and those using catalytic oxidation as the end treatment unit (mainly absorption - catalytic oxidation technology, Abs.-C.O.). So, this paper determines there

*Corresponding author's e-mail: lijing@wti.ac.cn 
are totally 5 technical solutions on vapor recovery to be evaluated, i.e. $\mathrm{m}=5$, and a decision solution set $\mathrm{U}=\left\{\mathrm{u}_{1}, \mathrm{u}_{2}, \mathrm{u}_{3}, \mathrm{u}_{4}, \mathrm{u}_{5}\right\}$, of which $u_{1}, u_{2}, \ldots u_{5}$ represent the 5 different technical solutions.

2.1.2 Factor Index Set. Suppose the solutions to be evaluated are described with $\mathrm{n}$ factor indexes, and its factor index set is $\mathrm{V}=\left\{V_{1}, V_{2}, \ldots V_{n}\right\}$. Since terminal companies operate for the sake of profit, factors in multiple aspects such as economy, safety and environmental protection shall be considering when choosing indexes. By analyzing influence factors to technology applications of vapor recovery, finally, 9 factor indexes are determined, i.e. $\mathrm{n}=9$, including the cost of construction and operation, energy consumption, treatment efficiency and capacity, annual recovery volume, life time, floor space, safety and environmental impact of the unit, which are expressed by $V_{1}, V_{2}, \ldots V_{9}$ respectively, denoted as the factor index set $\mathrm{V}=\left\{V_{1}, V_{2}, \ldots V_{9}\right\}$.
2.1.3 Evaluation matrix. In the solution $u_{i}$, the value of the index $V_{j}$ is expressed by $u_{i j}=(i=1,2, \ldots m ; j=1,2, \ldots n)$, and from this, the evaluation matrix obtained for the solution set $\mathrm{U}$ to the index set $\mathrm{V}$ is $E=\left(u_{i j}\right)_{m \times n}$.

2.1.4 Decision matrix. Factor indexes may be generally divided into benefit indexes (the higher the better), cost indexes (the less the better) and medium indexes (the closer to a fixed value the better) [3]. Of factor indexes influencing the technical solutions to vapor recovery for refined oil terminals, the cost of construction and installation, energy consumption, floor space and environmental impact are cost indexes while the treatment efficiency and capacity, annual recovery rate, life time and safety are benefit indexes.

Suppose the relatively ideal decision solution $u_{0}=\left(u_{01}, u_{02}, \ldots u_{0 j}\right)$ which satisfies:

$$
\mathrm{u}_{0 j}=\left\{\begin{array}{l}
\max \left(u_{1 j}, u_{2 j}, \ldots u_{m j}\right), \text { when } \mathrm{v}_{j} \text { is a benefit index, } \\
\min \left(u_{1 j}, u_{2 j}, \ldots u_{m j}\right), \text { when } \mathrm{v}_{j} \text { is a cost index, } \mathrm{j}=1,2,3, \ldots \mathrm{n} \\
M \operatorname{Manu}_{i j}=\frac{1}{m} \sum_{i=1}^{m} u_{i j}, \text { when } \mathrm{v}_{j} \text { is a medium index, }
\end{array}\right.
$$

From this, the decision matrix of the solution set $\mathrm{U}$ versus the index set $\mathrm{V}$ is obtained:

$$
D=\left(u_{i j}\right)_{(m+1) \times n}(\mathrm{i}=1,2, \ldots \mathrm{m} ; \mathrm{j}=1,2, \ldots \mathrm{n}) .
$$

\subsection{Normalization of Decision Matrix}

Since the values of different indexes vary in dimension and the order of magnitude, raw data must be normalized before making the solution decision, i.e. divide each figure in the sequence by the first figure of it, to get a new sequence to eliminate the incommensurability caused by dimensions and their units so that all indexes can be compared.

Suppose the process of the data normalization is INGO: $u_{i j}=u_{i j}$, and satisfy:

$$
u_{i j}^{\prime}=\left\{\begin{array}{l}
\frac{u_{i j}}{u_{0 j}}, \text { when } \mathrm{v}_{j} \text { is a benefit index, } \\
\frac{u_{0 j}}{u_{i j}}, \text { when } \mathrm{v}_{j} \text { is a cost index, } \mathrm{j}=1,2,3, \ldots \mathrm{n} \\
\frac{\min \left(u_{i j}, u_{0 j}\right)}{\max \left(u_{i j}, u_{0 j}\right)}, \text { when } \mathrm{v}_{j} \text { is a medium index, }
\end{array}\right.
$$

The matrix $D^{\prime}=\left(u_{i j}^{\prime}\right)_{(m+1) \times n}$ is called the normalized matrix of the matrix $D=\left(u_{i j}\right)_{(m+1) \times n}$. Obviously, after initialization,

$\mathrm{u}_{0 j}^{\prime}=1(j=1,2, \ldots n)$, and

$u_{0}^{\prime}=\left(u_{01}^{\prime}, u_{02}^{\prime}, \ldots . u_{0 n}^{\prime}\right)=(1,1, \ldots 1)$ is a

relatively ideal solution.

\subsection{Grey Correlation Degree (GCD) of Solutions}

The GCD of solutions refer to the measure of the correlation between the factor indexes of two solutions to be evaluated that change over time or from different objects [4]. Of the ideal solution $u_{0}$ is denoted by the vector $u_{0}^{\prime}=\left(u_{01}^{\prime}, u_{02}^{\prime}, \ldots u_{0 n}^{\prime}\right)=(1,1, \ldots 1)$ and the solution to be evaluated $u_{i}$ is denoted by the vector $u_{i}^{\prime}=\left(u_{i 1}^{\prime}, u_{i 2}^{\prime}, \ldots u_{i n}^{\prime}\right)$, the GCD between the factor index $V_{j}$ of the solution to be evaluated $u_{i}$ and the one $V_{j}$ of the ideal solution $u_{0}$ is:

$$
r_{i j}=\frac{\min _{1 \leq i \leq m} \min _{1 \leq j \leq n}\left|\dot{u}_{i j}^{\prime}-u_{0 j}^{\prime}\right|+\rho \max _{1 \leq i \leq m} \max _{1 \leq j \leq n}\left|u_{i j}^{\prime}-u_{0 j}^{\prime}\right|}{\left|u_{i j}^{\prime}-u_{0 j}^{\prime}\right|+\rho \max _{1 \leq i \leq m} \max _{1 \leq j \leq n}\left|u_{i j}-u_{0 j}^{\prime}\right|}
$$


Wherein:

$r_{i j}$ is called the correlation coefficient, reflecting the closeness between two sequences compared at a moment;

$\rho$ is called the resolution coefficient, $\rho \in(0,1)$, and $\rho=0.5$ is usually artificially selected.

\subsection{Determination of Factor Indexes Weights}

There are many methods for determining index weights, including expert consultation, multi-analysis, subjective experience and analytic hierarchy process (AHP) and so on. Using the weighted least-square method of AHP[5], this paper first makes pairwise comparison on factor indexes $a_{j}=(j=1,2, \ldots n)$ of technical solutions on vapor recovery via expert consultation, and based on the compared result, determine the judgment

$$
\begin{gathered}
I=(1,1, \ldots 1)^{T} \\
B=\left[\begin{array}{c}
\left(\sum_{i=1}^{n} a_{i 1}^{2}+n-2 a_{11}\right) \\
-\left(a_{21}+a_{12}\right) \\
\vdots \\
-\left(a_{n 1}+a_{1 n}\right)
\end{array}\right.
\end{gathered}
$$$$
\left.\begin{array}{ccc}
-\left(a_{12}+a_{21}\right) & \ldots & -\left(a_{1 n}+a_{n 1}\right) \\
\left(\sum_{i=1}^{n} a_{i 2}^{2}+n-2 a_{22}\right) & \ldots & -\left(a_{2 n}+a_{n 2}\right) \\
\vdots & \ddots & \vdots \\
-\left(a_{n 2}+a_{2 n}\right) & \ldots & \left(\sum_{i-1}^{n} a_{i n}^{2}+n-2 a_{n n}\right)
\end{array}\right] .
$$

matrix $\mathrm{A}=\left(a_{i j}\right)_{n \times n}$ :$$
A=\left[\begin{array}{cccc}
\mathrm{a}_{11} & \mathrm{a}_{12} & \cdots & \mathrm{a}_{1 n} \\
\mathrm{a}_{21} & \mathrm{a}_{22} & \cdots & \mathrm{a}_{2 n} \\
\vdots & \vdots & \ddots & \vdots \\
\mathrm{a}_{n 1} & \mathrm{a}_{n 2} & \cdots & \mathrm{a}_{n n}
\end{array}\right]
$$

Suppose the weight vector of $\mathrm{n}$ evaluation indexes $a_{1}, a_{2}, \ldots a_{n}$ relative to the total target is $\mathrm{W}=\left(\omega_{1}, \omega_{2}, \ldots \omega_{n}\right)$, apply the weighted least-square method to get:

$$
B \omega+\lambda 1=0
$$

Wherein:
Solve the formula (5) and (6) to get:

$$
\vec{\omega}=\frac{B^{-1} e}{e^{T} B^{-1} e}
$$

Wherein:

$\vec{\omega}$ is the weight vector of the judgment matrix $\mathrm{A}=\left(a_{i j}\right)_{n \times n}$.

With the formula (7), it is convenient to calculate the weight coefficients $\omega$ of all factor indexes so as to get the weight vector $\mathrm{W}$.

\subsection{Calculation of Weighted GCD}

It is known from the above analysis that the matrix comprised of $\mathrm{m} \times \mathrm{n} \quad \mathrm{GC} \quad$ coefficients $r_{i j}(i=0,1,2, \ldots m ; j=1,2, \ldots n)$ is a multitarget GC matrix: $\mathrm{R}=\left(r_{i j}\right)_{m \times n}$. To facilitate the comparison, the correlation coefficients are weighted and centralized to get the weighted GCD [6]:

$$
r_{i}=\sum_{j}^{n} \omega_{j} r_{i j}, i=1,2, \ldots m
$$

Wherein, $r_{i}$ is the weighted correlation between each solution and the ideal solution, i.e. similarity, while $\omega_{j}$ is the weight coefficient of the index $V_{j}$.

\subsection{Decision Model}

It is known from the above analysis that, the weighted GCDs between each technical solution $u_{i}$ and the relatively ideal solution $u_{0}$ constitute the GC vector $\mathrm{R}=\left(r_{1}, r_{2}, \ldots r_{m}\right)$, and according to the GCD decision theory, the weighted GCD of solutions can be used directly as the criteria to evaluate whether a solution is good or not[7], that is:

(1) The bigger the value of $r_{i}$ is, it means the higher similarity between the solution $u_{i}$ and the ideal solution $u_{0}$, the higher the priority.

(2) If $r_{i}=\max \left(r_{1}, r_{2}, \ldots r_{m}\right)$, the solution $u_{i}$ is the most optimal.

(3) Sequencing by the size of $r_{i}$, accordingly the priority arrangement of solutions is obtained.

\section{Examples of Practical Applications}

\begin{tabular}{|c|c|c|c|c|c|c|c|c|c|}
\hline $\begin{array}{l}\text { Technical } \\
\text { solution }\end{array}$ & $\begin{array}{c}\text { Cost (RMB } \\
10^{4} \text { Yuan) }\end{array}$ & $\begin{array}{c}\text { Energy } \\
\text { consumptio } \\
\mathrm{n}\left(\mathrm{kwh} / \mathrm{m}^{3}\right)\end{array}$ & $\begin{array}{c}\text { Efficiency } \\
, \%\end{array}$ & $\begin{array}{c}\text { Capacity, } \\
\mathrm{m}^{3} / \mathrm{h}\end{array}$ & $\begin{array}{c}\text { Annual } \\
\text { recovery, } \mathrm{t}\end{array}$ & $\begin{array}{l}\text { Life } \\
\text { time }\end{array}$ & $\begin{array}{c}\text { Floor } \\
\text { space, } \\
\mathrm{m}^{2}\end{array}$ & $\begin{array}{l}\text { Safety } \\
, \%\end{array}$ & $\begin{array}{c}\text { Environme } \\
\text { ntal } \\
\text { impact, } \%\end{array}$ \\
\hline
\end{tabular}

It is planned to build a vapor recovery device at a refined oil terminal of which values of factor indexes of 5 technical solutions to be evaluated are shown in Table 1 .

Table 1. Values of factor indexes of 5 vapor recovery technical solutions 


\begin{tabular}{llccccccccc}
\hline Ads.-Abs. & 810 & 0.7 & 97 & 1250 & 1050 & 25 & 208 & 85 & 90 \\
Con.-Ads. & 805 & 0.73 & 97 & 1100 & 986 & 25 & 128 & 80 & 90 \\
Abs.-M.S. & 920 & 0.83 & 98 & 800 & 991 & 20 & 150 & 90 & 85 \\
Con.-M.S. & 915 & 0.88 & 98 & 720 & 820 & 20 & 80 & 90 & 87.5 \\
Abs.-C.O. & 665 & 0.9 & 99 & 1000 & 650 & 20 & 150 & 95 & 82.5 \\
\hline
\end{tabular}

(1) Establishment of Decision Matrix

Based on data in Table 1, the index set of the relatively ideal technical solution is obtained:

$\mathrm{u}_{0}=\left(c_{5}, 0.7,99,1250,1050,25,80,95,82.5\right)$

Based on Formula (1), the decision matrix of solution set $\mathrm{U}$ versus index set $\mathrm{V}$ is obtained: $\mathrm{D}=\left(u_{i j}\right)_{(m+1) \times n}$.

$$
D=\left[\begin{array}{lllllllll}
810 & 0.7 & 97 & 1250 & 1050 & 25 & 208 & 85 & 90 \\
805 & 0.73 & 97 & 1100 & 98 \zeta & 25 & 128 & 80 & 90 \\
920 & 0.83 & 98 & 800 & 991 & 20 & 150 & 90 & 85 \\
915 & 0.88 & 98 & 720 & 820 & 20 & 80 & 90 & 87.5 \\
65 & 0.9 & 99 & 1000 & 550 & 20 & 90 & 95 & 82.5 \\
c \zeta 5 & 0.7 & 99 & 1250 & 1050 & 25 & 80 & 95 & 82.5
\end{array}\right]
$$

(2) Normalization of Index Matrix

Since different indexes have different dimensions, to eliminate their impact on the decision results, the formula (2) is used to normalize the matrix $D$ to get the normalization matrix $\mathrm{D}^{\prime}=\left(u_{i j}^{\prime}\right)_{(m+1) \times n}$.

$$
D^{\prime}=\left[\begin{array}{lllllllll}
0.821 & 1 & 0.9798 & 1 & 1 & 1 & 0.3846 & 0.8947 & 0.9167 \\
0.8251 & 0.9589 & 0.9798 & 0.88 & 0.939 & 1 & 0.525 & 0.8421 & 0.9167 \\
0.7228 & 0.8434 & 0.9899 & 0.54 & 0.9438 & 0.8 & 0.5333 & 0.9474 & 0.970 \zeta \\
0.7258 & 0.7955 & 0.9899 & 0.576 & 0.781 & 0.8 & 1 & 0.9474 & 0.9429 \\
1 & 0.7778 & 1 & 0.8 & 0.619 & 0.8 & 0.8889 & 1 & 1 \\
1 & 1 & 1 & 1 & 1 & 1 & 1 & 1 & 1
\end{array}\right]
$$

(3) Determination of Factor Indexes Weights

The judgment matrix A of the weights of factor indexes of technical solutions of the terminal company is obtained by its technical and managerial staff making

$$
A=\left[\begin{array}{lllllllll}
1 & 5 & 1 & 1 / 5 & 1 / 5 & 1 / 3 & 1 / 3 & 1 / 5 & 1 / 5 \\
1 / 5 & 1 & 1 / 4 & 1 & 1 / 3 & 1 / 3 & 1 / 3 & 1 / 5 & 1 / 5 \\
1 & 4 & 1 & 1 & 3 & 2 & 1 / 2 & 1 / 5 & 1 / 5 \\
5 & 1 & 1 & 1 & 3 & 2 & 1 / 2 & 1 / 5 & 1 / 5 \\
5 & 3 & 1 & 1 / 3 & 1 & 1 & 1 / 2 & 1 / 5 & 1 / 5 \\
3 & 1 / 3 & 1 / 2 & 1 / 2 & 1 & 1 & 2 & 1 / 5 & 1 / 5 \\
3 & 3 & 2 & 2 & 2 & 1 / 2 & 1 & 1 / 3 & 1 / 3 \\
5 & 5 & 5 & 5 & 5 & 5 & 3 & 1 & 1 \\
5 & 5 & 5 & 5 & 5 & 5 & 3 & 1 & 1
\end{array}\right]
$$

Using the formula (5) to operate the judgment matrix A to get:

$$
A=\left[\begin{array}{lllllllll}
34.38 & -5.20 & -2.00 & -5.20 & -5.20 & -3.33 & -3.33 & -5.20 & -5.20 \\
-5.20 & 9.52 & -4.25 & -2.00 & -3.33 & -3.33 & -3.33 & -5.20 & -5.20 \\
-2.00 & -4.25 & 39.33 & -2.00 & -3.33 & -2.50 & -2.50 & -5.20 & -5.20 \\
-5.20 & -2.00 & -2.00 & 48.33 & -3.33 & -2.50 & -2.50 & -5.20 & -5.20 \\
-5.20 & -3.33 & -2.00 & -3.33 & 44.44 & -2.00 & -2.50 & -5.20 & -5.20 \\
-3.33 & -3.33 & -2.50 & -2.50 & -2.00 & 22.59 & -2.50 & -5.20 & -5.20 \\
-3.33 & -3.33 & -2.50 & -2.50 & -2.50 & -2.50 & 38.47 & -3.33 & -3.33 \\
-5.20 & -5.20 & -5.20 & -5.20 & -5.20 & -5.20 & -3.33 & 158.00 & -2.00 \\
-5.20 & -5.20 & -5.20 & -5.20 & -5.20 & -5.20 & -3.33 & -2.00 & 158.00
\end{array}\right]
$$

Then, based on formula (6) and (7), using the weighted least-square method to get the weight vector $\mathrm{W}$ of the factor index.

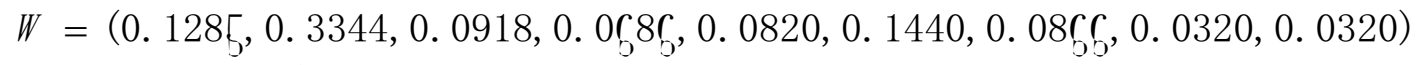

(4) Determination of correlation coefficient pairwise comparison and scoring between every 2 of 9 indexes in the technical solutions and making statistic on the compared results. 
Operate $u_{0 j}^{\prime}-u_{i j}^{\prime}$ against the matrix $\mathrm{D}^{\prime}$ to get:

$$
\bar{S}=\left[\begin{array}{rrrrrrrrr}
0.179 & 0 & 0.0202 & 0 & 0 & 0 & 0.5154 & 0.1053 & 0.0833 \\
0.1739 & 0.0411 & 0.0202 & 0.12 & 0.051 & 0 & 0.375 & 0.1579 & 0.0833 \\
0.2772 & 0.156 \varnothing & 0.0101 & 0.36 & 0.0562 & 0.2 & 0.4567 & 0.0526 & 0.0294 \\
0.2732 & 0.2045 & 0.0101 & 0.4240 & 0.2190 & 0.2 & 0 & 0.0526 & 0.0571 \\
0 & 0.2222 & 0 & 0.2 & 0.381 & 0.2 & 0.1111 & 0 & 0
\end{array}\right]
$$

From the matrix $\bar{S}$, the following is obtained:

$$
\begin{gathered}
\max _{1 \leq i \leq m} \max _{1 \leq j \leq n}\left|u_{i j}^{\prime}-u_{0 j}^{\prime}\right|=0.52 \\
\min _{1 \leq i \leq m} \min _{1 \leq j \leq n}\left|u_{i j}^{\prime}-u_{0 j}^{\prime}\right|=0
\end{gathered}
$$

Substitute this result and the resolution coefficient $\rho=0.5$ into the formula (3), to get the expression of the correlation coefficient:

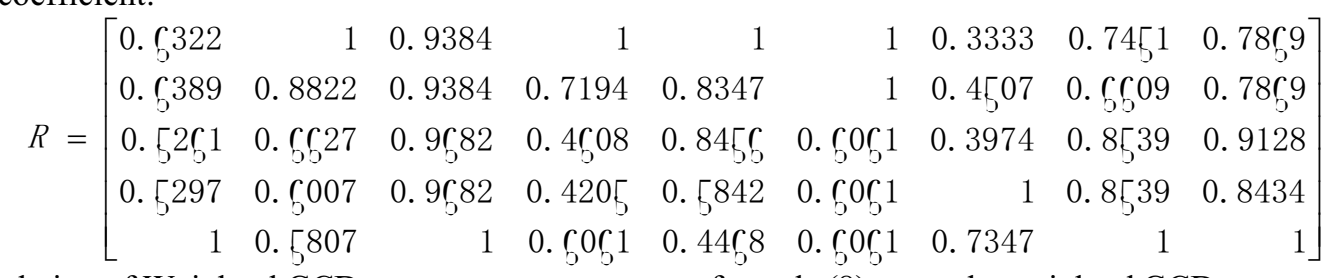

(5) Calculation of Weighted GCD

formula (8) to get the weighted GCD vectors $r$ of 5 vapor recovery technical solutions for the refined oil terminal.

Substituted the calculated weight vector $\mathrm{W}$ of factor index and the correlation coefficient matrix $\mathrm{R}$ into the

$$
r=\left(r_{1}, r_{2}, r_{3}, r_{4}, r_{5}\right)=(0.8743,0.810\lceil, 0.6573,0.6528,0.7077)
$$

As a result, the weighted GCDs corresponding to 5 technical solutions are shown in Table 2 .

Table 2. Weighted GCDs of 5 technical solutions

\begin{tabular}{cccccc}
\hline $\begin{array}{c}\text { Solutions to be } \\
\text { evaluated }\end{array}$ & Ads.- Abs. & Con.-Ads. & Abs.-M.S. & Con.-M.S. & Abs.-C.O. \\
\hline Weighted GCD: $r$ & 0.8743 & 0.8105 & 0.6573 & 0.6628 & 0.7077 \\
\hline
\end{tabular}

It is known from Table 2 that the weighted GCDs sequence of 5 vapor recovery technical solutions is $u_{1}>u_{2}>u_{5}>u_{4}>u_{3}$. According to the GCD decision theory, for this refined oil terminal, the most optimal vapor recovery technical solution is adsorption absorption, followed by condensation - adsorption, and absorption - membrane is lower in GCD, so its adaptability is poorer.

\section{Conclusion}

Aiming at the complexity of multi-target decision problems in technical solutions to vapor recovery at refined oil terminals, this paper provides a scientific decision method for selecting the best ones by building a GCD-based multi-target decision model, using the weighted least-square method of AHP to determine the index weight, using the weighted GCDs of technical solutions as judgment criteria, and finally demonstrating its viability and effectiveness with an example of practical application.

\section{Acknowledgments}

Thanks are due to members of the project team for their excellent technical supports and Director Rongchang Chen for his strict review of the manuscript. We also acknowledge the editors and the anonymous reviewers for insightful suggestions on this article.

\section{References}

1. www.gov.cn, (2015) Law of the People's Republic of China on Prevention and Control of Atmospheric Pollution (Presidential Decree No.21). http://www.gov.cn/zhengce/201508/30/content_2922326.htm.

2. Li X H,(2007) Grey-correlation-based Multi-target Decision Model and Its Application. Journal of Shandong University (Natural Science), 42(12):3335 .

3. Tian R, Sun L F, Li B Y, Liao Z W.(2013) Roughfuzzy-set-based TOPSIS Supplier Evaluation Method. Application Research Of Computers, 30(8):2319-2322. 
4. Wang G Y, Niu Z G, Wang M, Shen J J.(2011) Grey-correlation TOPSIS for Evaluation of Foundation Treatment Schemes. Mathematics in Practice and Theory, 41(9):115-120.

5. Sun R S, Hu Z, Wang L, Huangpu G G.(2016) Study on Factors Affecting Fatigue of Maintenance Personnel Based on Least Square Method for Weights and Entropy Weight Method. Safety and Environmental Engineering, 23(3):167-170.

6. Song J P, Dong D W, Gao G A.(2002) Study on Solution Decision Model Based on AHP and Grey Correlation. Journal of Southwest Jiaotong University, 8(4)464-466.

7. Yue D D, Liang H T, Zhao L, Wang T H.(2016) Application of AHP-based Grey-Correlation Decision Model in Solution Optimization. Water Technology, 10(4):29-34. 